\title{
A partition theorem for $\alpha$-large sets
}

\author{
by \\ Teresa Bigorajska and Henryk Kot larski (Siedlce)
}

\begin{abstract}
Working with Hardy hierarchy and the notion of largeness determined by it, we define the notion of a partition of a finite set of natural numbers $A=\cup_{i<m} A_{i}$ being $\alpha$-large and show that for ordinals $\alpha, \beta<\varepsilon_{0}$ satisfying suitable assumptions, if $A$ is $\left(\omega^{\beta} \cdot \alpha\right)$-large and is partitioned as above and the partition itself is not $\alpha$-large, then at least one $A_{i}$ is $\omega^{\beta}$-large.
\end{abstract}

The goal of this paper is to work out a combinatorial result which generalizes one of the results of Ketonen-Solovay [5]. Working below the ordinal $\varepsilon_{0}$ we define the notion of a partition $A=\cup_{i<m} A_{i}$ (where $A \subseteq \omega$ ) being $\alpha$-large and show that (under suitable assumptions on $\alpha$ and $\beta$ ), if $A$ is $\left(\omega^{\beta} \cdot \alpha\right)$-large and the partition itself is not $\alpha$-large then there exists an $\omega^{\beta}$ large homogeneous set. Of course, our paper heavily depends on the work of Ketonen-Solovay [5]. Indeed, from a point of view we generalize one of their results ([5], Theorem 4.7) from $\omega^{2}$ to $\varepsilon_{0}$. We would like to point out that when working with the so-called Hardy hierarchy we are highly influenced by the work of Z. Ratajczyk (see [9], [6], [7] and his final [10]). It should be noticed that the idea of Hardy hierarchy was developed by several schools (see, e.g., [3] and [2]).

Let $h$ be a finite increasing function (in the usual sense of the word, that is, $\forall x, y \in \operatorname{Dom}(h)[x<y \Rightarrow f(x)<f(y)])$. Assume moreover that $\forall x x<h(x)$. For every $\alpha<\varepsilon_{0}$ we define a function $h_{\alpha}$, by induction on $\alpha$. We put $h_{0}(x)=x$ and $h_{\alpha+1}(x)=h_{\alpha}(h(x))$.

Before defining the limit step we need to define, for each limit $\lambda<\varepsilon_{0}$, a sequence $\{\lambda\}(n)$ of ordinals convergent to $\lambda$ from below. We put $\{\omega\}(n)=n$, and, more generally, $\left\{\omega^{\alpha+1}\right\}(n)=\omega^{\alpha} \cdot n$. For limit $\gamma$ we put $\left\{\omega^{\gamma}\right\}(n)=$ $\omega^{\{\gamma\}(n)}$. Finally,

1991 Mathematics Subject Classification: Primary 05A18; Secondary 03F99.

Research of H. Kotlarski was supported in part by The State Committee for Scientific Research (Poland), KBN, grant number 2 P03A 07110. 
$\left\{\omega^{\alpha_{0}} \cdot m_{0}+\ldots+\omega^{\alpha_{s}} \cdot m_{s}\right\}(n)=\omega^{\alpha_{0}} \cdot m_{0}+\ldots+\omega^{\alpha_{s}} \cdot\left(m_{s}-1\right)+\left\{\omega^{\alpha_{s}}\right\}(n)$, where $\lambda=\omega^{\alpha_{0}} \cdot m_{0}+\ldots+\omega^{\alpha_{s}} \cdot m_{s}$ is the Cantor normal form expansion of $\lambda$, i.e., $\alpha_{0}>\ldots>\alpha_{s}$. It is easy to see that these conditions determine exactly one sequence $\{\{\lambda\}(n): n\}$, for each $\lambda<\varepsilon_{0}$. Observe also that Ketonen and Solovay [5] use a slightly different notion of $\{\lambda\}(n)$. We shall call the sequence $\{\lambda\}(n)$ the fundamental sequence for $\lambda$. It is possible to extend fundamental sequences to larger ordinals (cf. e.g. [8]). For example let $\omega_{0}=\omega$ and $\omega_{n+1}=\omega^{\omega_{n}}$. Then $\left\{\varepsilon_{0}\right\}(n)=\omega_{n}$ is a fundamental sequence for $\varepsilon_{0}$.

Now we are ready to define $h_{\lambda}$ for $\lambda$ limit. We simply put $h_{\lambda}(x)=$ $h_{\{\lambda\}(x)}(x)$. The sequence $h_{\alpha}: \alpha<\varepsilon_{0}$ is called the Hardy hierarchy based on $h$.

This notion allows us to define a set $A$ of natural numbers to be $\alpha$-large. Namely, $A$ is $\alpha$-large iff $\left(h^{A}\right)_{\alpha}(a)$ is defined, where $h^{A}$ denotes the successor in the sense of $A$ (i.e., the function with domain $A \backslash\{\max A\}$ which associates with every $b$ in its domain the next element of $A$ ) and $a=\min A$. We shall write just $h$ if the meaning of $A$ is clear from the context. One can restate this definition of largeness in the following manner. A set $A$ is 0-large iff it is nonempty. $A$ is $(\alpha+1)$-large iff $A \backslash\{\min A\}$ is $\alpha$-large. $A$ is $\lambda$-large, $\lambda$ limit, iff it is $\{\lambda\}(\min A)$-large. Observe that Ketonen and Solovay [5] use a slightly different notion of largeness.

Let $A$ be a finite subset of $\omega$. We say that the partition $A=\cup_{0 \leq i \leq e} B_{i}$ of $A$ is $\alpha$-large if the set $E=\left\{\min B_{0}, \ldots, \min B_{e}\right\}$ is $\alpha$-large. A set or partition which is not $\alpha$-large will be called $\alpha$-small.

For ordinals $\alpha, \beta, \gamma<\varepsilon_{0}$ we write $\alpha \rightarrow(\beta)_{\gamma}^{1}$ if for every $\alpha$-large set $A$ with $\min A>0$ and every partition $A=\cup_{0 \leq i \leq e} B_{i}$ of $A$ which is $\gamma$-small, there exists $i \leq e$ such that $B_{i}$ is $\beta$-large. We keep the superscript 1 in the above notation just to follow the usual notation in Ramsey theory (cf. [4]).

For every $\alpha<\varepsilon_{0}$ let $\operatorname{LM}(\alpha)$ denote the greatest (i.e., leftmost) exponent in the Cantor normal form expansion of $\alpha$. By $\varrho(\alpha)$ we mean the smallest (i.e., rightmost) exponent of $\alpha$. We write $\beta \gg \alpha$ if either $\alpha=0$ or $\beta=0$ or all the exponents in the Cantor normal form of $\beta$ are $\geq$ all the exponents in the normal form of $\alpha$, i.e., $\varrho(\beta) \geq \operatorname{LM}(\alpha)$. Observe that $\beta \gg \alpha$ does not imply $\beta \geq \alpha$, indeed, $0 \gg \alpha$ for each $\alpha$ and $\omega^{4} \gg \omega^{4} \cdot 3$. We should remark that if the relation $\beta \gg \alpha$ holds then the Cantor normal form of $\beta+\alpha$ is just the concatenation of the Cantor normal forms of $\beta$ and $\alpha$.

The main result of this paper is as follows (we needed it as the main combinatorial lemma in [1]).

Theorem 1. If $\alpha, \beta<\varepsilon_{0}, \alpha \geq 1$ and $\beta \gg \operatorname{LM}(\alpha)$ then $\omega^{\beta} \cdot \alpha \rightarrow\left(\omega^{\beta}\right)_{\alpha}^{1}$.

We shall need several other notions. We extend the notion of a fundamental sequence to nonlimit ordinals by putting $\{0\}(n)=0$ and $\{\alpha+1\}(n)=\alpha$. 
For $\beta, \alpha<\varepsilon_{0}$ we write $\beta \rightarrow_{n} \alpha$ iff there exists a finite sequence $\alpha_{0}, \ldots, \alpha_{k}$ of ordinals such that $\alpha_{0}=\beta, \alpha_{k}=\alpha$ and for every $m<k$ there exists $j_{m} \leq n$ such that $\alpha_{k+1}=\left\{\alpha_{k}\right\}\left(j_{m}\right)$. We write $\beta \Rightarrow_{n} \alpha$ if there exists a sequence as above, but with each $j_{m}=n$. Observe that both relations $\rightarrow_{n}, \Rightarrow_{n}$ are transitive and imply $\beta \geq \alpha$.

Lemma 2. (i) For every $\alpha, b, \alpha \Rightarrow_{b} 0$.

(ii) If $\beta \gg \alpha$ and $\alpha \Rightarrow_{n} \gamma$ then $\beta+\alpha \Rightarrow_{n} \beta+\gamma$.

(iii) If $k<l$ and $n>0$ then $\omega^{\alpha} \cdot l \Rightarrow_{n} \omega^{\alpha} \cdot k$.

(iv) If $\beta \Rightarrow_{n} \alpha$ and $n>0$ then $\omega^{\beta} \Rightarrow_{n} \omega^{\alpha}$.

(v) $\alpha \Rightarrow_{n}\{\alpha\}(j)$ and $\{\alpha\}(n) \Rightarrow_{n}\{\alpha\}(j)$ for $j \leq n$.

(vi) $\{\alpha\}(n) \Rightarrow_{1}\{\alpha\}(j)$ for $0<j \leq n$.

(vii) If $n \leq b$ and $\alpha \Rightarrow_{n} \beta$ then $\alpha \Rightarrow_{b} \beta$.

(viii) $\beta \Rightarrow_{n} \alpha$ iff $\beta \rightarrow_{n} \alpha$.

(ix) If $\alpha<\beta$ then there exists b such that $\beta \Rightarrow_{b} \alpha$.

Proof. See Ketonen-Solovay [5]. As pointed out above, they work with slightly different fundamental sequences, but their proofs work in our case as well. In fact, (i)-(viii) are not very difficult to prove (in the order as stated); the proof of the last claim (by induction on $\beta$ ) uses (vii).

Lemma 3. Let $\lambda$ be a limit ordinal smaller than $\varepsilon_{0}$. Then if $\beta \gg \operatorname{LM}(\lambda)$ then for every $n \in \omega,\left\{\omega^{\beta} \cdot \lambda\right\}(n)=\omega^{\beta} \cdot\{\lambda\}(n)$.

Proof. Let $\lambda$ be limit and let $\varrho$ be the smallest exponent in the Cantor normal form expansion of $\lambda$. Then $\lambda=\delta+\omega^{\varrho}$ for some $\delta \gg \omega^{\varrho}$. Let $\beta \gg$ $\operatorname{LM}(\lambda)$ and $n \in \omega$. We have

$$
\left\{\omega^{\beta} \cdot \lambda\right\}(n)=\left\{\omega^{\beta}\left(\delta+\omega^{\varrho}\right)\right\}(n)=\left\{\omega^{\beta} \cdot \delta+\omega^{\beta+\varrho}\right\}(n)=\omega^{\beta} \cdot \delta+\left\{\omega^{\beta+\varrho}\right\}(n) .
$$

The last equality holds because $\omega^{\beta} \cdot \delta \gg \omega^{\beta+\varrho}$. Obviously $\beta \gg \varrho$, hence if $\varrho=\alpha+1$ for some $\alpha$ then

$$
\begin{aligned}
\omega^{\beta} \cdot \delta+\left\{\omega^{\beta+\varrho}\right\}(n) & =\omega^{\beta} \cdot \delta+\left\{\omega^{\beta+\alpha+1}\right\}(n)=\omega^{\beta} \cdot \delta+\omega^{\beta+\alpha} \cdot n \\
& =\omega^{\beta} \cdot\left(\delta+\omega^{\alpha} \cdot n\right)=\omega^{\beta} \cdot\left(\delta+\left\{\omega^{\alpha+1}\right\}(n)\right) \\
& =\omega^{\beta} \cdot\left\{\delta+\omega^{\varrho}\right\}(n)=\omega^{\beta} \cdot\{\lambda\}(n) .
\end{aligned}
$$

Let $\varrho$ be limit. By the assumption $\beta \gg \varrho$ we get

$$
\begin{aligned}
\omega^{\beta} \cdot \delta+\left\{\omega^{\beta+\varrho}\right\}(n) & =\omega^{\beta} \cdot \delta+\omega^{\{\beta+\varrho\}(n)}=\omega^{\beta} \cdot \delta+\omega^{\beta+\{\varrho\}(n)} \\
& =\omega^{\beta} \cdot\left(\delta+\omega^{\{\varrho\}(n)}\right)=\omega^{\beta} \cdot\left(\delta+\left\{\omega^{\varrho}\right\}(n)\right) \\
& =\omega^{\beta} \cdot\{\lambda\}(n) .
\end{aligned}
$$


LEMma 4. Let $h$ be a function as above. Then for every $\alpha<\varepsilon_{0}$ :

(i) $h_{\alpha}$ is increasing.

(ii) For every $\beta, b$ if $\alpha \Rightarrow_{b} \beta$ then if $h_{\alpha}(b)$ exists then $h_{\beta}(b)$ exists and $h_{\alpha}(b) \geq h_{\beta}(b)$.

Pr o of. By simultaneous induction on $\alpha$, left to the reader.

Below if we write $A=\left\{a_{0}, \ldots, a_{\text {card } A-1}\right\}$ we assume that this enumeration is the natural one, i.e., in increasing order.

Lemma 5. (i) For every $\alpha$ if $A, B$ are finite sets of the same cardinality and such that for every $i<\operatorname{card} A, b_{i} \leq a_{i}$ then for every $i<\operatorname{card} A$ if $\left(h^{A}\right)_{\alpha}\left(a_{i}\right)$ exists then $\left(h^{B}\right)_{\alpha}\left(b_{i}\right)$ exists and $\left(h^{A}\right)_{\alpha}\left(a_{i}\right) \geq\left(h^{B}\right)_{\alpha}\left(b_{i}\right)$.

(ii) If $A, B$ are finite sets, $A$ is $\alpha$-large, $\operatorname{card} A=\operatorname{card} B$ and for every $i<\operatorname{card} A, b_{i} \leq a_{i}$ then $B$ is $\alpha$-large.

(iii) If $A \subseteq B$ and $A$ is $\alpha$-large then $B$ is $\alpha$-large.

$\operatorname{Proof}$. The first part is immediate by induction on $\alpha$, the second is a direct consequence of the first one. The third part follows from the observation that if $A \subseteq B$ then $B$ has an initial segment of cardinality card $A$. But obviously, if a set has an $\alpha$-large initial segment then it is $\alpha$-large itself, so the second part may be applied.

The following is a minor variant of Lemma 5 in which we speak of sets of different cardinalities. We write $h_{\alpha}(x) \downarrow$ rather than " $h_{\alpha}(x)$ exists".

Lemma 6. For every $\alpha$ and every $D, E$, if $D \subseteq E, x \in D$ and $\left(h^{D}\right)_{\alpha}(x) \downarrow$ then $\left(h^{E}\right)_{\alpha}(x) \downarrow$ and $\left(h^{E}\right)_{\alpha}(x) \leq\left(h^{D}\right)_{\alpha}(x)$.

Proof (by induction on $\alpha$ ). If $\alpha=0$ the conclusion is obvious. Assume the conclusion for $\alpha$; we derive it for $\alpha+1$. So let $D, E$ satisfy the assumption. Let $x \in D$ be such that $\left(h^{D}\right)_{\alpha+1}(x)$ exists. Then $\left(h^{D}\right)_{\alpha+1}(x)=$ $\left(h^{D}\right)_{\alpha}\left(\left(h^{D}\right)(x)\right)$. Let $y=\left(h^{D}\right)(x)$. We apply the inductive assumption to $y$. Thus we infer $\left(h^{E}\right)_{\alpha}(y) \downarrow$ and $\left(h^{E}\right)_{\alpha}(y) \leq\left(h^{D}\right)_{\alpha}(y)$. But $\left(h^{E}\right)(x) \leq$ $\left(h^{D}\right)(x)=y$, hence $\left(h^{E}\right)_{\alpha+1}(x)=\left(h^{E}\right)_{\alpha}\left(\left(h^{E}\right)(x)\right) \leq\left(h^{E}\right)_{\alpha}\left(\left(h^{D}\right)(x)\right) \leq$ $\left(h^{D}\right)_{\alpha}\left(\left(h^{D}\right)(x)\right)=\left(h^{D}\right)_{\alpha+1}(x)$ because $\left(h^{E}\right)_{\alpha}$ is increasing by Lemma 4 . We leave the limit step to the reader.

Lemma 7. Let $h$ be as above. Then for every $\alpha$ and every $\beta \gg \alpha, h_{\beta+\alpha}=$ $h_{\beta} \circ h_{\alpha}$.

Proof. By induction on $\alpha$.

Let us restate this fact in the following manner.

Lemma 8. Let $A$ be a finite set and let $\beta \gg \alpha$. Then $A$ is $(\beta+\alpha)$-large iff there exists $u \in A$ such that $\{x \in A: x \leq u\}$ is $\alpha$-large and $\{x \in A: u \leq x\}$ is $\beta$-large. 
We shall need one more idea (once again, known from Ketonen-Solovay [5]). For every $\alpha<\varepsilon_{0}$ we define the norm of $\alpha,\|\alpha\|$, in the following manner. We let $\|0\|=0$. If $\alpha>0$ we write $\alpha=\omega^{\alpha_{0}} \cdot a_{0}+\ldots+\omega^{\alpha_{r}} \cdot a_{r}$ in the Cantor normal form and let $\|\alpha\|=\sum_{i=0}^{r} a_{i} \cdot\left(1+\left\|\alpha_{i}\right\|\right)$. The following fact strengthens Lemma 2(ix) so that $b$ may be chosen to depend only on $\alpha$.

Lemma 9. (i) For every $\alpha<\varepsilon_{0}$ if $a \geq\|\alpha\|$ then for every $\beta>\alpha$ we have $\beta \Rightarrow_{a} \alpha$ and hence $\{\beta\}(a) \geq \alpha$.

(ii) For every $\alpha$ and every $\beta$, if $\varrho(\beta)>\alpha$ and $a=\|\alpha\|$ then $\{\beta\}(a) \gg \omega^{\alpha}$ and $\{\beta\}(a)+\omega^{\alpha}<\beta$.

Proof. For (i) see Ketonen-Solovay [5]. We prove (ii). We write $\beta=$ $\delta+\omega^{\beta^{\prime}}$ where $\delta \gg \omega^{\beta^{\prime}}$. By the assumption, $\beta^{\prime}>\alpha$. If $\beta^{\prime}=\beta^{\prime \prime}+1$ then $\{\beta\}(a)=\delta+\omega^{\beta^{\prime \prime}} \cdot a$ with $\beta^{\prime \prime} \geq \alpha$, so the first conclusion is immediate. The second one follows from the fact that in the decisive step the exponent $\beta^{\prime}$ was changed to the smaller one, i.e. $\beta^{\prime \prime}$. If $\beta^{\prime}$ is limit then $\left\{\delta+\omega^{\beta^{\prime}}\right\}(a)=$ $\delta+\omega^{\left\{\beta^{\prime}\right\}(a)}$. By (i), $\left\{\beta^{\prime}\right\}(a) \geq \alpha$, so the first conclusion holds. The second does as well because in the decisive step the exponent $\beta^{\prime}$ was lowered to $\left\{\beta^{\prime}\right\}(a)$.

We shall need an additional lemma.

LEMMA 10. (i) $\forall \gamma>0 \forall b>0 \gamma \Rightarrow_{b} 1$.

(ii) $\forall \alpha \gg \omega \forall u>b>1\{\alpha\}(u) \Rightarrow_{b}\{\alpha\}(b)+1$.

(iii) $\forall \alpha \gg \omega \forall \delta \gg \omega^{\alpha} \forall u>b>1 \delta+\omega^{\{\alpha\}(u)} \Rightarrow_{b} \delta+\omega^{\{\alpha\}(b)} \cdot b$.

(iv) If a set $D$ is $\left(\delta+\omega^{\{\alpha\}(u)}\right)$-large and $b=\min D$ satisfies $u>b>1$ then $D$ is $\left(\delta+\omega^{\{\alpha\}(b)} \cdot b\right)$-large.

Proof. (i) is immediate by induction on $\gamma$. (ii) is proved by induction on $\alpha$, the cases $\alpha=\omega$ and $\alpha \rightarrow \alpha+\omega$ being immediate, so we show only the step $\alpha \gg \omega^{2}$. Write $\alpha=\delta+\omega^{\tau}$, where $\delta \gg \omega^{\tau}$. Thus, $\tau>1$. If $\tau=\varrho+1$ then

$$
\{\alpha\}(u)=\left\{\delta+\omega^{\varrho+1}\right\}(u)=\delta+\omega^{\varrho} \cdot u=\delta+\omega^{\varrho} \cdot b+\omega^{\varrho} \cdot(u-b) .
$$

We use (i) to infer $\{\alpha\}(u) \Rightarrow_{b}\{\alpha\}(b)+1$ as required. So let $\tau$ be limit. Then $\{\tau\}(u) \Rightarrow_{b}\{\tau\}(b)+1$ by the inductive assumption, so by Lemma 2(iii),

$$
\{\alpha\}(u)=\left\{\delta+\omega^{\tau}\right\}(u)=\delta+\omega^{\{\tau\}(u)} \Rightarrow_{b} \delta+\omega^{\{\tau\}(b)+1} .
$$

Moreover, $\omega^{\{\tau\}(b)+1} \Rightarrow_{b} \omega^{\{\tau\}(b)} \cdot b=\omega^{\{\tau\}(b)}+\omega^{\{\tau\}(b)} \cdot(b-1)$ and the same argument as above works.

(iii) follows from (ii) and Lemma 2(iii).

In order to prove part (iv), let $D, u, b$ satisfy the assumption. That is, we have $h_{\delta+\left\{\omega^{\alpha}\right\}(u)}(\min D) \downarrow$. By (iii) and Lemma $4, h_{\delta+\left\{\omega^{\alpha}\right\}(b) \cdot b}(\min D) \downarrow$ as required.

The main lemma needed for the proof of Theorem 1 is as follows. 
Lemma 11. For every $\alpha$, every $\beta \gg \omega^{\alpha}$ and every $A, B$, if $\min A>0$ and $A$ is $\left(\beta+\omega^{\alpha}\right)$-large, $B \subseteq A$ and $B$ is $\omega^{\alpha}$-small then $A \backslash B$ is $\beta$-large.

Proof. Let $T(\beta, \alpha)$ be the following property:

for every $A, B$, if $A$ is $\left(\beta+\omega^{\alpha}\right)$-large and $B \subseteq A$ is $\omega^{\alpha}$-small then $A \backslash B$ is $\beta$-large

and we shall prove the statement $\forall \alpha \forall \beta \gg \omega^{\alpha} T(\beta, \alpha)$ by induction on $\alpha$.

CASE $\alpha=0$. Then $A$ is $\left(\beta+\omega^{0}\right)$-large, i.e. $(\beta+1)$-large, and $B$ is 1 -small. If $\beta=0$ then $A$ is 1-large, i.e., has at least two elements, but $B$ being 1 -small has at most one element, so $A \backslash B$ is nonempty, so 0-large. If $\beta>0$ then $A$ is $(\beta+1)$-large so $A \backslash\left\{a_{0}\right\}$ is $\beta$-large. Also, $B$ being 1 -small has at most one element. It follows that $A \backslash\left\{a_{0}\right\}$ and $A \backslash B$ satisfy the assumption of Lemma 5 (these sets have the same cardinality and the $i$ th element of $A \backslash B$ is $\leq$ the $i$ th element of $A \backslash\left\{a_{0}\right\}$ ), hence $A \backslash B$ is $\beta$-large.

CASE $\alpha=1$. Exactly as above, the case $\beta=0$ is obvious. For other cases we proceed by induction on $\beta$.

Let $\beta=\omega$. So let $A$ be $(\omega+\omega)$-large and let $B \subseteq A$ be $\omega$-small. Let $u=\left(h^{A}\right)_{\omega}\left(a_{0}\right)$.

Case 1: $b_{0}>u$. Then $\{x \in A: x \leq u\} \subseteq A \backslash B$. The first of these sets is $\omega$-large, so the second is as well by Lemma 5 .

Case 2: $b_{0}=u$. Then there exists $z \in A \backslash B$ with $z>u$ (otherwise $\{x \in A: u \leq x\} \subseteq B$, so $B$ is $\omega$-large by Lemma 5 ). It follows that $\{x \in A: x<u\} \cup\{z\} \subseteq A \backslash B$, so this set is $\omega$-large, again by Lemma 5 .

Case 3: $a_{0}<b_{0}<u$. In order to show that $A \backslash B$ is $\omega$-large it suffices to show that it has more than $a_{0}$ elements, indeed, $\min (A \backslash B)=a_{0}$. But $A$ has more than $a_{0}+u$ elements and $B$, being $\omega$-small, has at most $b_{0}<u$ elements.

Case 4: $a_{0}=b_{0}$. Then $B$ has at most $a_{0}$ elements, so $A \backslash B$ has more than $u$ elements. If $\min (A \backslash B) \leq u$ then we are done. Otherwise $\{x \in A$ : $x \leq u\} \subseteq B$, so this set is $\omega$-large, contrary to assumption.

Assume $T(\beta, 1)$; we prove $T(\beta+\omega, 1)$. So let $A$ be $(\beta+\omega+\omega)$-large and let $B \subseteq A$ be $\omega$-small. Let $u=\left(h^{A}\right)_{\omega}\left(a_{0}\right)$ and $w=\left(h^{A}\right)_{\omega}(u)$.

Case 1: $b_{0}>u$. Let $A^{\prime}=A \backslash\{x \in A: x<u\}$. Thus $B \subseteq A^{\prime}$. By $T(\beta, 1)$, $A^{\prime} \backslash B$ is $\beta$-large, hence $\{x \in A: x<u\} \cup\left(A^{\prime} \backslash B\right)=A \backslash B$ is $(\beta+\omega)$-large by Lemma 8 .

Case 2: $b_{0}=u$. Let $A^{\prime}$ be $\{x \in A: u \leq x\}$. By $T(\beta, 1)$, the set $C=A^{\prime} \backslash B$ is $\beta$-large. Let $c_{0}$ be, as usual, the smallest element of $C$. Then $A \backslash B=\{x \in$ $A: x<u\} \cup C=\left(\{x \in A: x<u\} \cup\left\{c_{0}\right\}\right) \cup C$ is $(\beta+\omega)$-large by Lemma 8 .

Case 3: $a_{0}<b_{0}<u$. Obviously, $B$ has at most $b_{0}$ elements (otherwise it is $\omega$-large), so $B$ has less than $u$ elements. Let $k=\operatorname{card}(\{x \in A: w<x\})$. Thus, $A \backslash B$ has at least $a_{0}+k+1$ elements. Let $c_{0}=a_{0}, c_{1}, \ldots, c_{a_{0}}$ be 
the list of the first $a_{0}+1$ elements of $A \backslash B$ in increasing order. We claim that $c_{a_{0}} \leq w$. For otherwise there are at least $k$ elements of $A \backslash B$ which are $>c_{a_{0}}>w$. But this is impossible, as there are only $k-1$ such elements of $A$. Let $E=\left\{e_{0}, \ldots, e_{k}\right\}$ be the set of the $k+1$ consecutive elements of $A \backslash B$, beginning with $e_{0}=c_{a_{0}}$. Then $E$ is $\beta$-large, indeed, its cardinality is $k+1$ and its elements are $\leq$ the corresponding elements of $\{x \in A: w \leq x\}$. It follows that $A \backslash B$ contains $\left\{c_{0}, \ldots, c_{a_{0}}\right\} \cup E$, so it is $(\beta+\omega)$-large by Lemma 8.

Case $4: b_{0}=a_{0}$. Then $B$, being $\omega$-small, has at most $a_{0}$ elements, hence $A \backslash B$ has more than $u+k$ elements. Let $E=\left\{e_{0}, \ldots, e_{u+k}\right\}$ be the set of the first $u+k+1$ of them. Then $E$ is $(\beta+\omega)$-large because its elements are $\leq$ the corresponding elements of $\{x \in A: u \leq x\}$.

Assume $\forall \beta^{\prime}<\beta T\left(\beta^{\prime}, 1\right)$ and $\varrho(\beta)>1$; we check $T(\beta, 1)$. So let $A$ be $(\beta+\omega)$-large. Let $u=\left(h^{A}\right)_{\omega}\left(a_{0}\right)$ as usual, so $A=\{x \in A: x \leq$ $u\} \cup\{x \in A: u \leq x\}$. The first of these sets is $\omega$-large and the second one is $\beta$-large, i.e., $\{\beta\}(u)$-large. As $\varrho(\beta)>1$ we have (i) $\{\beta\}(u) \gg \omega$ and (ii) $\{\beta\}(u)+\omega<\beta$. Let $B$ be an $\omega$-small subset of $A$. The set $A$ is $(\{\beta\}(u)+\omega)$-large and by $T(\{\beta\}(u), 1), A \backslash B$ is $\{\beta\}(u)$-large. Observe that $\min (A \backslash B)=c_{0} \leq u$, for otherwise $\{x \in A: x \leq u\} \subseteq B$, so $B$ is $\omega$-large contrary to assumption. If $c_{0}=u$ then obviously $A \backslash B$ is $\beta$-large, so assume that $c_{0}<u$. By Lemma 2(vi), $\{\beta\}(u) \Rightarrow_{1}\{\beta\}\left(c_{0}\right)$, so $\{\beta\}(u) \Rightarrow_{c_{0}}\{\beta\}\left(c_{0}\right)$ by (vii) of the same lemma. By Lemma $4,\left(h^{A \backslash B}\right)_{\{\beta\}\left(c_{0}\right)}\left(c_{0}\right)$ exists, so $A \backslash B$ is $\beta$-large.

We show the nonlimit step in the proof of Lemma 11, i.e.,

$$
\forall \alpha\left[\left(\forall \beta \gg \omega^{\alpha} T(\beta, \alpha)\right) \Rightarrow\left(\forall \beta \gg \omega^{\alpha+1} T(\beta, \alpha+1)\right)\right] .
$$

Once again, the case $\beta=0$ is obvious. Indeed, if $A$ is $\omega^{\alpha+1}$-large and $B$ is its $\omega^{\alpha+1}$-small subset, then $A \backslash B$ is nonempty, so 0-large.

CASE $\beta=\omega^{\alpha+1}$. Let $A$ be $\left(\omega^{\alpha+1}+\omega^{\alpha+1}\right)$-large. Let $u=\left(h^{A}\right)_{\omega^{\alpha+1}}\left(a_{0}\right)$. Then $A=\{x \in A: x \leq u\} \cup\{x \in A: u \leq x\}$ and both of these sets are $\omega^{\alpha+1}$-large. Let $B$ be an $\omega^{\alpha+1}$-small subset of $A$.

Case 1: $b_{0}>u$. Then $\{x \in A: x \leq u\}$ is contained in $A \backslash B$, so this set is $\omega^{\alpha+1}$-large.

Case 2: $b_{0}=u$. Then there exists $z \in A \backslash B$ with $z>u$ (otherwise $\{x \in A: u \leq x\} \subseteq B$ and hence $B$ is $\omega^{\alpha+1}$-large, which contradicts the assumption), so $\{x \in A: x<u\} \cup\{z\}$ is contained in $A \backslash B$, so this set is $\omega^{\alpha+1}$-large.

Case 3: $a_{0}<b_{0}<u$. We let $c_{0}=b_{0}=\min B$ and $c_{i+1}=\left(h^{B}\right)_{\omega^{\alpha}}\left(c_{i}\right)$. This induction breaks after $r$ steps, where $r \leq b_{0}$, otherwise $B$ is $\omega^{\alpha+1}$-large. That is, the last $c_{i}$ is $c_{r-1}$. We let $A_{0}=A$ and $A_{i+1}=A_{i} \backslash\left\{x \in B: c_{i} \leq x<\right.$ $\left.c_{i+1}\right\}$ and $A_{r}=A_{r-1} \backslash\left\{x \in B: c_{r-1} \leq x\right\}$. Observe that $A_{0}$ is $\left(\omega^{\alpha} \cdot\left(a_{0}+u\right)\right)$ - 
large, and (by the inductive assumption), $A_{i}$ is $\left(\omega^{\alpha} \cdot\left(a_{0}+u-i\right)\right)$-large. In particular, $A_{r}$ is $\left(\omega^{\alpha} \cdot a_{0}\right)$-large, i.e., $\omega^{\alpha+1}$-large, indeed $a_{0}=\min (A \backslash B)$.

Case $4: b_{0}=a_{0}$. Arguing as in case 3 we see that $A \backslash B$ is $\left(\omega^{\alpha} \cdot u\right)$-large. Thus if $\min (A \backslash B)=u$ this set is $\omega^{\alpha+1}$-large. If $d=\min (A \backslash B)<u$ then $\left\{\omega^{\alpha+1}\right\}(u) \Rightarrow_{1}\left\{\omega^{\alpha+1}\right\}(d)$ by Lemma 2 (vi), hence $\omega^{\alpha} \cdot u \Rightarrow_{d} \omega^{\alpha} \cdot d$ by (vii) of the same lemma. By Lemma $4,\left(h^{A \backslash B}\right)_{\omega^{\alpha} \cdot d}(d)$ exists (because $\left(h^{A \backslash B}\right)_{\omega^{\alpha} \cdot u}(d)$ exists).

We prove the implication $T(\beta, \alpha+1) \Rightarrow T\left(\beta+\omega^{\alpha+1}, \alpha+1\right)$ for $\beta \gg \omega^{\alpha+1}$. So let $A$ be $\left(\beta+\omega^{\alpha+1} \cdot 2\right)$-large and let $B$ be its $\omega^{\alpha+1}$-small subset. Let $u=\left(h^{A}\right)_{\omega^{\alpha+1}}\left(a_{0}\right)$ and $w=\left(h^{A}\right)_{\omega^{\alpha+1}}(u)$.

Case 1: $b_{0} \geq u$. Let $A^{\prime}=\{x \in A: x \geq u\}$. Then $B \subseteq A^{\prime}$. By $T(\beta, \alpha+1)$, $A^{\prime} \backslash B$ is $\beta$-large, hence $A \backslash B=\left(\{x \in A: x<u\} \cup\left\{c_{0}\right\}\right) \cup\left(A^{\prime} \backslash B\right)$, where $c_{0}=\min \left(A^{\prime} \backslash B\right)$, is $\left(\beta+\omega^{\alpha+1}\right)$-large.

Case 2: $a_{0}<b_{0}<u$. We put $d_{0}=b_{0}=\min B$ and $d_{i+1}=\left(h^{B}\right)_{\omega^{\alpha}}\left(d_{i}\right)$. Let $r$ be the greatest $i$ such that $d_{i}$ exists. We must have $r<b_{0}$ for otherwise $B$ would be $\omega^{\alpha+1}$-large. Let $D_{i}=\left\{x \in B: d_{i} \leq x<d_{i+1}\right\}$ and $D_{r}=\{x \in$ $\left.B: d_{r} \leq x\right\}$. Observe that none of these sets is $\omega^{\alpha}$-large. On the other hand, $A$ is $\left(\beta+\omega^{\alpha}\left(u+a_{0}\right)\right)$-large. It follows that $A \backslash D_{0}$ is $\left(\beta+\omega^{\alpha} \cdot\left(u+a_{0}-1\right)\right)$-large, etc., $A \backslash B=A \backslash \cup_{i \leq r} D_{i}$ is $\left(\beta+\omega^{\alpha} \cdot\left(u+a_{0}-r\right)\right)$-large. But $r+1 \leq u$, hence $A \backslash B$ is $\left(\beta+\omega^{\alpha} \cdot a_{0}\right)$-large, so it is $\left(\beta+\omega^{\alpha+1}\right)$-large because its minimum is $a_{0}$.

Case 3: $b_{0}=a_{0}$. Exactly as above, by subtracting $B$ from $A$ in parts which are not $\omega^{\alpha}$-large we derive that $A \backslash B$ is $\left(\beta+\omega^{\alpha} \cdot u\right)$-large. Indeed, there are only $a_{0}$ parts as above because $\min B=a_{0}$ and this set is $\omega^{\alpha+1}$-small. If $\min (A \backslash B)=u$ then we are done. Otherwise $e=\min (A \backslash B)<u$. But $\omega^{\alpha} \cdot u \Rightarrow_{1} \omega^{\alpha} \cdot e$ by Lemma $2(\mathrm{vi})$, and hence $\omega^{\alpha} \cdot u \Rightarrow_{e} \omega^{\alpha} \cdot e$ by (vii) of the same lemma. By Lemma $4,\left(h^{A \backslash B}\right)_{\omega^{\alpha} \cdot e}(e)$ exists because $\left(h^{A \backslash B}\right)_{\omega^{\alpha} \cdot u}(e)$ exists.

Thus in order to prove the nonlimit step $\alpha+1$ in the proof of Lemma 11 it remains to check the case $\varrho(\beta)>\alpha+1$. So let $\varrho(\beta)>\alpha+1$ and assume that for all $\beta^{\prime}<\beta, T\left(\beta^{\prime}, \alpha+1\right)$ holds. Let $A$ be $\left(\beta+\omega^{\alpha+1}\right)$-large and let $B$ be its $\omega^{\alpha+1}$-small subset. As usual, we let $u=\left(h^{A}\right)_{\omega^{\alpha+1}}\left(a_{0}\right)$, so that $A=\{x \in A: x \leq u\} \cup\{x \in A: u \leq x\}$; the first of these sets is $\omega^{\alpha+1}$ large, the second being $\beta$-large. It follows that $A$ is $\left(\{\beta\}(u)+\omega^{\alpha+1}\right)$-large. Observe that $u=\left(h^{A}\right)_{\omega^{\alpha+1}}\left(a_{0}\right) \geq\|\alpha+1\|$. By Lemma 9, $\{\beta\}(u) \gg \omega^{\alpha+1}$ and $\{\beta\}(u)+\omega^{\alpha+1}<\beta$. By $T(\{\beta\}(u), \alpha+1), A \backslash B$ is $\{\beta\}(u)$-large. Observe that $\min (A \backslash B)=c \leq u$, otherwise $\{x \in A: x \leq u\} \subseteq B$, so $B$ is $\omega^{\alpha+1}$-large, contrary to assumption. If $c=u$ then we are done, $A \backslash B$ is $\{\beta\}(\min (A \backslash B))$ large. So assume that $c<u$. Then $\left(h^{A \backslash B}\right)_{\{\beta\}(u)}(c) \downarrow$. Also we have $\{\beta\}(u) \Rightarrow_{1}$ $\{\beta\}(c)$ by Lemma 2(vi), hence, by Lemma 2(vii), $\{\beta\}(u) \Rightarrow_{c}\{\beta\}(c)$. By Lemma $4,\left(h^{A \backslash B}\right)_{\{\beta\}(c)}(c) \downarrow$ and $A \backslash B$ is $\beta$-large. 
CASE $\alpha$ limit. So, by assumption we have $\forall \alpha^{\prime}<\alpha \forall \beta \gg \omega^{\alpha^{\prime}} T\left(\beta, \alpha^{\prime}\right)$; we want to prove $\forall \beta \gg \omega^{\alpha} T(\beta, \alpha)$. As usual, the case $\beta=0$ is obvious.

Let $\beta=\omega^{\alpha}$. Let $A$ be $\left(\omega^{\alpha}+\omega^{\alpha}\right)$-large and let $B$ be its $\omega^{\alpha}$-small subset. As usual, let $u=\left(h^{A}\right)_{\omega^{\alpha}}\left(a_{0}\right)$.

Case 1: $b_{0}>u$. Then $\{x \in A: x \leq u\} \subseteq A \backslash B$, so this set is $\omega^{\alpha}$-large as required.

Case 2: $b_{0}=u$. Then there exists $z>u$ with $z \in A \backslash B$, for otherwise $\{x \in A: u \leq x\} \subseteq B$, so $B$ is $\omega^{\alpha}$-large contrary to assumption. Thus $\{x \in A: x<u\} \cup\{z\} \subseteq A \backslash B$ and $A \backslash B$ is $\omega^{\alpha}$-large.

Case 3: $a_{0}<b_{0}<u$ (the main case). Let $D=\left\{x \in A: b_{0} \leq x\right\}$ and let $E=\{x \in A: u \leq x\}$. Then $E$ is $\omega^{\alpha}$-large, i.e. it is $\omega^{\{\alpha\}(u)_{-}}$ large. It follows that $D$ is $\omega^{\{\alpha\}(u)}$-large, indeed, it contains $E$. By Lemma 10 (iv), $D$ is $\left(\left\{\omega^{\alpha}\right\}\left(b_{0}\right) \cdot b_{0}\right)$-large, in particular, it is $\left(\left\{\omega^{\alpha}\right\}\left(b_{0}\right)+\left\{\omega^{\alpha}\right\}\left(b_{0}\right)\right)$ large (reason: $a_{0}<b_{0}$, hence $b_{0}>1$ ). We apply the inductive assumption $T\left(\left\{\omega^{\alpha}\right\}\left(b_{0}\right),\{\alpha\}\left(b_{0}\right)\right)$ and infer that $D \backslash B$ is $\left\{\omega^{\alpha}\right\}\left(b_{0}\right)$-large. By Lemma 5(iii), $A \backslash B$ is $\left\{\omega^{\alpha}\right\}\left(b_{0}\right)$-large. We also have $\left\{\omega^{\alpha}\right\}\left(b_{0}\right) \Rightarrow_{a_{0}}\left\{\omega^{\alpha}\right\}\left(a_{0}\right)$ by Lemma 2(vii), hence $A \backslash B$ is $\left\{\omega^{\alpha}\right\}\left(a_{0}\right)$-large, i.e., $\omega^{\alpha}$-large.

Case $4: b_{0}=a_{0}$. In this case $B$ is $\left\{\omega^{\alpha}\right\}\left(a_{0}\right)$-small. But $A$ is $\left(\left\{\omega^{\alpha}\right\}(u)+\right.$ $\left.\left\{\omega^{\alpha}\right\}\left(a_{0}\right)\right)$-large. By the inductive assumption $T\left(\left\{\omega^{\alpha}\right\}(u),\{\alpha\}\left(a_{0}\right)\right), A \backslash B$ is $\left\{\omega^{\alpha}\right\}(u)$-large. Let $s=\min (A \backslash B)$. If $s=u$ then we are done. If $s<u$ then $\left\{\omega^{\alpha}\right\}(u) \Rightarrow_{s}\left\{\omega^{\alpha}\right\}(s)$, hence $A \backslash B$ is $\left\{\omega^{\alpha}\right\}(s)$-large, i.e., $\omega^{\alpha}$-large. The case $s>u$ cannot happen, for if it does then $\{x \in A: x \leq u\} \subseteq B$, so $B$ is $\omega^{\alpha}$-large, contrary to assumption.

Assume $T(\beta, \alpha)$, where $\beta \gg \omega^{\alpha}$; we prove $T\left(\beta+\omega^{\alpha}, \alpha\right)$. So let a set $A$ be $\left(\beta+\omega^{\alpha}+\omega^{\alpha}\right)$-large and let $B$ be its $\omega^{\alpha}$-small subset. Let $u, w$ be as before, i.e., $u=\left(h^{A}\right)_{\omega^{\alpha}}\left(a_{0}\right)$ and $w=\left(h^{A}\right)_{\omega^{\alpha}}(u)$.

Case 1: $b_{0} \geq u$. Then $B \subseteq\{x \in A: u \leq x\}$ and by the inductive assumption $T(\beta, \alpha),\{x \in A \backslash B: u \leq x\}$ is $\beta$-large. It follows that $A \backslash B=$ $\{x \in A: x \leq u\} \cup\{x \in A \backslash B: u \leq x\}$ is $\left(\beta+\omega^{\alpha}\right)$-large.

Case 2: $a_{0}<b_{0}<u$. Let $E=\{x \in A: u \leq x\}$ and $D=\{x \in A$ : $\left.b_{0} \leq x\right\}$. Then $E$ is $\left(\beta+\omega^{\alpha}\right)$-large, hence it is $\left(\beta+\left\{\omega^{\alpha}\right\}(u)\right)$-large. It follows that $D$ is $\left(\beta+\left\{\omega^{\alpha}\right\}(u)\right)$-large as well. Exactly as above, it follows that $D$ is $\left(\beta+\left\{\omega^{\alpha}\right\}\left(b_{0}\right) \cdot b_{0}\right)$-large, hence it is $\left(\beta+\left\{\omega^{\alpha}\right\}\left(b_{0}\right) \cdot 2\right)$-large. By the inductive assumption $T\left(\beta+\left\{\omega^{\alpha}\right\}\left(b_{0}\right),\{\alpha\}\left(b_{0}\right)\right), D \backslash B$ is $\left(\beta+\left\{\omega^{\alpha}\right\}\left(b_{0}\right)\right)$-large, i.e. $\left(\beta+\omega^{\alpha}\right)$-large. Hence $A \backslash B$ is $\left(\beta+\omega^{\alpha}\right)$-large as a superset of $D \backslash B$.

Case 3: $b_{0}=a_{0}$. Then $B$ is $\left\{\omega^{\alpha}\right\}\left(a_{0}\right)$-small. By the inductive assumption $T\left(\beta+\omega^{\alpha}+\left\{\omega^{\alpha}\right\}\left(a_{0}\right),\{\alpha\}\left(a_{0}\right)\right), A \backslash B$ is $\left(\beta+\omega^{\alpha}\right)$-large.

Finally, let $\varrho(\beta)>\alpha$. Let, as usual, $A$ be $\left(\beta+\omega^{\alpha}\right)$-large and let $B$ be its $\omega^{\alpha}$-small subset. Let also $u=\left(h^{A}\right)_{\omega^{\alpha}}\left(a_{0}\right)$. Clearly $u \geq\|\alpha+1\|$, hence $A$ is $\left(\{\beta\}(u)+\omega^{\alpha}\right)$-large. By Lemma $9,\{\beta\}(u) \gg \omega^{\alpha}$ and $\{\beta\}(u)+\omega^{\alpha}<\beta$. By the inductive assumption $T(\{\beta\}(u), \alpha), A \backslash B$ is $\{\beta\}(u)$-large. Let $s=\min (A \backslash B)$. 
Exactly as above, $s \leq u$ for otherwise $\{x \in A: x \leq u\} \subseteq B$, so $B$ is $\omega^{\alpha}$-large contrary to assumption. If $s=u$ then we are done. Otherwise, $s<u$, hence $\{\beta\}(u) \Rightarrow_{s}\{\beta\}(s)$, so $A \backslash B$ is $\{\beta\}(s)$-large, i.e. $\beta$-large.

It should be noticed that Lemma 11 admits a generalization in which we speak not only about ordinals of the form $\omega^{\alpha}$. It is as follows.

Theorem 12. For every $\alpha$ and $\beta \gg \alpha$ and every $A, B$, if $A$ is $\beta+\alpha$-large, $B \subseteq A$ and $B$ is $\alpha$-small, then $A \backslash B$ is $\beta$-large.

Proof. Let $A$ be $(\beta+\alpha)$-large where $\beta \gg \alpha$, and let $B$ be its $\alpha$-small subset. Write $\alpha=\omega^{\alpha_{s}}+\ldots+\omega^{\alpha_{0}}$, where $\alpha_{s} \geq \ldots \geq \alpha_{0}$. Let $e=\max \{i \leq s$ : $B$ is $\left(\omega^{\alpha_{i}}+\ldots+\omega^{\alpha_{0}}\right)$-large $\}$. Let $h$ denote the successor in the sense of $B$. Let $B_{0}=\left\{x \in B: x<h_{\omega^{\alpha_{0}}}(\min B)\right\}, B_{i+1}=\left\{x \in B: h_{\omega^{\alpha_{i}}}\left(\min B_{i}\right) \leq\right.$ $\left.x<h_{\omega^{\alpha_{i+1}}}\left(h_{\omega^{\alpha_{i}}}\left(\min B_{i}\right)\right)\right\}$ for $i<e$. We let $B_{e+1}=B \backslash \cup_{0 \leq i \leq e} B_{i}$. Then $B=\cup_{0 \leq i \leq e+1} B_{i}$. Observe that no $B_{i}, i \leq e+1$, is $\omega^{\alpha_{i}}$-large. By Lemma 11, by induction on $i$, we infer that $A \backslash\left(B_{0} \cup \ldots \cup B_{i}\right)$ is $\left(\beta+\omega^{\alpha_{s}}+\ldots+\omega^{\alpha_{i+1}}\right)$ large. It follows that $A \backslash B$ is $\beta$-large.

Proof of Theorem 1. By induction on $\alpha$. The case $\alpha=1$ is obvious, indeed, if a partition is 1-small then there is only one part.

Assume the conclusion holds for $\alpha$; we derive it for $\alpha+1$. Let $A$ be an $\left(\omega^{\beta} \cdot(\alpha+1)\right)$-large subset of $\omega$ and let $A=\cup_{0 \leq i \leq e} B_{i}$ be an $(\alpha+1)$-small partition of $A$. Let $E=\left\{\min B_{0}, \ldots, \min B_{e}\right\}$, so $E$ is $(\alpha+1)$-small. We may assume that $\min E=\min B_{0}$. We put $C=A \backslash B_{0}$. If $B_{0}$ is $\omega^{\beta}$-small then by Lemma 11, $C$ is $\left(\omega^{\beta} \cdot \alpha\right)$-large. Consider the partition $C=\cup_{1 \leq i \leq e} B_{i}$ of $C$. Let $E_{1}=\left\{\min B_{1}, \ldots, \min B_{e}\right\}$. But the partition of $A$ is $(\alpha+1)$-small, hence $h_{\alpha+1}\left(\min B_{0}\right) \uparrow$ (where $h$ denotes the successor in the sense of $E$ ). It follows that $h_{\alpha}\left(h\left(\min B_{0}\right)\right) \uparrow$. We have $h\left(\min B_{0}\right)=\min E_{1}$. Thus the above partition of $C$ is $\alpha$-small. We apply the inductive assumption to the set $C$ and the above-mentioned partition. Summing up, $B_{0}$ or at least one of $B_{i}, 1 \leq i \leq e$, is $\omega^{\beta}$-large.

Assume the conclusion for all ordinals smaller than $\lambda, \lambda$ limit. Let $A$ be an $\left(\omega^{\beta} \cdot \lambda\right)$-large set, where $\beta \gg \operatorname{LM}(\lambda)$. Let a partition $A=\cup_{0 \leq i \leq e} B_{i}$ be given and $\lambda$-small. Exactly as above, let $E=\left\{\min B_{0}, \ldots, \min B_{e}\right\}$. Then $A$ is $\left(\left\{\omega^{\beta} \cdot \lambda\right\}(\min A)\right)$-large. Thus $A$ is $\left(\omega^{\beta} \cdot\{\lambda\}(\min A)\right)$-large by Lemma 3 . Obviously, $E$ is $\{\lambda\}(\min A)$-small and $\beta \gg \operatorname{LM}(\{\lambda\}(\min A))$. By the inductive assumption, at least one of $B_{i}, i \leq e$, is $\omega^{\beta}$-large.

We show that the result of Theorem 1 is the best possible. Let $A$ be a finite subset of $\omega$, let $\beta<\varepsilon_{0}$ and let $A=\cup_{0 \leq i \leq e} B_{i}$ be the partition of $A$ determined by the following conditions:

(i) Each $B_{i}$ is of the form $A \cap[u, w]$ for some $u, w \in A$.

(ii) $\min B_{0}=\min A$ and for all $i=0, \ldots, e-1, \min B_{i+1}=h_{\omega^{\beta}}\left(\min B_{i}\right)$.

(iii) $h_{\omega^{\beta}}\left(\min B_{e}\right) \uparrow$. 
Of course, $h$ denotes the successor in the sense of $A$. Let, as usual, $E$ denote the set $\left\{\min B_{i}: i \leq e\right\}$. Let $H$ denote the successor in the sense of $E$. Obviously, $H(n)=h_{\omega^{\beta}}(n)$ for $n \in E \backslash\{\max E\}$. We show that

(*) if $A$ is $\left(\omega^{\beta} \cdot \alpha\right)$-large, where $\beta \gg \operatorname{LM}(\alpha)$, then $H_{\alpha}(\min A)=$ $h_{\omega^{\beta} \cdot \alpha}(\min A)$.

We prove $(*)$ by induction on $\alpha$, the steps $\alpha=0$ and $\alpha \rightarrow \alpha+1$ being evident. In the limit step one uses Lemma 3.

If $A$ is $\left(\omega^{\beta} \cdot(\alpha+1)\right)$-small, where $\beta \gg \varrho(\alpha)$, then by $(*)$ we infer immediately that the partition of $A$ determined by the above mentioned conditions is $(\alpha+1)$-small. But none of the sets $B_{i}$ is $\omega^{\beta}$-large.

\section{References}

[1] T. Bigorajska, H. Kotlarski and J. Schmerl, On regular interstices and selective types in countable arithmetically saturated models of Peano Arithmetic, Fund. Math. 158 (1998), 125-146.

[2] E. A. Cichon, A short proof of two recently discovered independence results using recursion theoretic methods, Proc. Amer. Math. Soc. 87 (1983), 704-706.

[3] M. V. H. Fairlough and S. S. W ainer, Ordinal complexity of recursive definitions, Inform. and Comput. 99 (1992), 123-153.

[4] R. Graham, B. Rothschild and J. Spencer, Ramsey Theory, 2nd ed., Wiley, 1990 .

[5] J. Ketonen and R. Solovay, Rapidly growing Ramsey functions, Ann. of Math. 113 (1981), 267-314.

[6] H. Kotlarski and Z. Ratajczyk, Inductive full satisfaction classes, Ann. Pure Appl. Logic 47 (1990), 199-223.

[7] - - - More on induction in the language with a satisfaction class, Z. Math. Logik 36 (1990), 441-454.

[8] W. Pohlers, Proof Theory, Lecture Notes in Math. 1047, Springer, 1989.

[9] Z. Ratajczyk, A combinatorial analysis of functions provably recursive in $I \Sigma_{n}$, Fund. Math. 130 (1988), 191-213.

[10] - Subsystems of true arithmetic and hierarchies of functions, Ann. Pure Appl. Logic 64 (1993), 95-152.

[11] R. Sommer, Transfinite induction within Peano arithmetic, ibid. 76 (1995), 231289 .

Institute of Mathematics

Agricultural and Pedagogical University (WSRP)

Orlicz-Dreszera 19/21

08-110 Siedlce, Poland

E-mail: hkl@impan.gov.pl terebi@wsrp.siedlce.pl

Received 27 January 1998;

in revised form 28 December 1998 